\title{
Physics-based Holo-Net for three-dimensional imaging
}

\author{
Ni Chen" ${ }^{\#}$ Yuqi Li ${ }^{\#}$ and Wolfgang Heidrich \\ Visual Computing Center, King Abdullah University of Science and Technology, Thuwal 23955, Saudi Arabia \\ wolfgang.heidrich@kaust.edu.sa
}

\begin{abstract}
We propose a physics-based holographic network (PBHolo-Net) for threedimensional imaging. Due to the involvement of the wavefield propagation, the network is efficient, stable, and can perform more precise hologram reconstruction. (C) 2020 The Author(s)
\end{abstract}

\section{Introduction}

Digital holography is a powerful tool for three-dimensional imaging since the volumetric information can be extracted from a single two-dimensional (2D) image, in which the optical wavefront is recorded [1]. Holographic volume reconstruction conventionally uses multiple diffraction calculations to obtain sectional reconstructed images from an in-line hologram, followed by using focus metrics. However, these methods are computationally intensive and require fine tuning parameters such as regularization and relaxation parameters [2,3]. Recently, machine learning has emerged as a prevailing tool in computational imaging. However, compared to other fields, machine learning has been under-utilized in digital holography [4]. Especially for 3D holographic imaging, only a few research has been reported for volumetric particle reconstruction [5,6]. The state-of-the-art technique can reconstruct either low concentration holograms $\left(1.9 \times 10^{-4}\right.$ to $6.1 \times 10^{-2}$ particle per pixel (ppp) $)$ with U-Net CNN [5] or relatively high concentration holograms $\left(1.9 \times 10^{-4}\right.$ to $\left.6.1 \times 10^{-2} \mathrm{ppp}\right)$ with time consuming preand post- hologram processing [6]. For the latter one, the reconstructed position of the particles shows an error of several voxels in depth. In this report, we propose a PBHolo-Net for 3D reconstruction from an in-line hologram. The forward and backward wavefield propagation between the 3D objects and the hologram is considered into the deep learning model, which makes the hologram reconstruction shows a precise focus position with acceptable noise rather than location error.

\section{The PBHolo-Net}
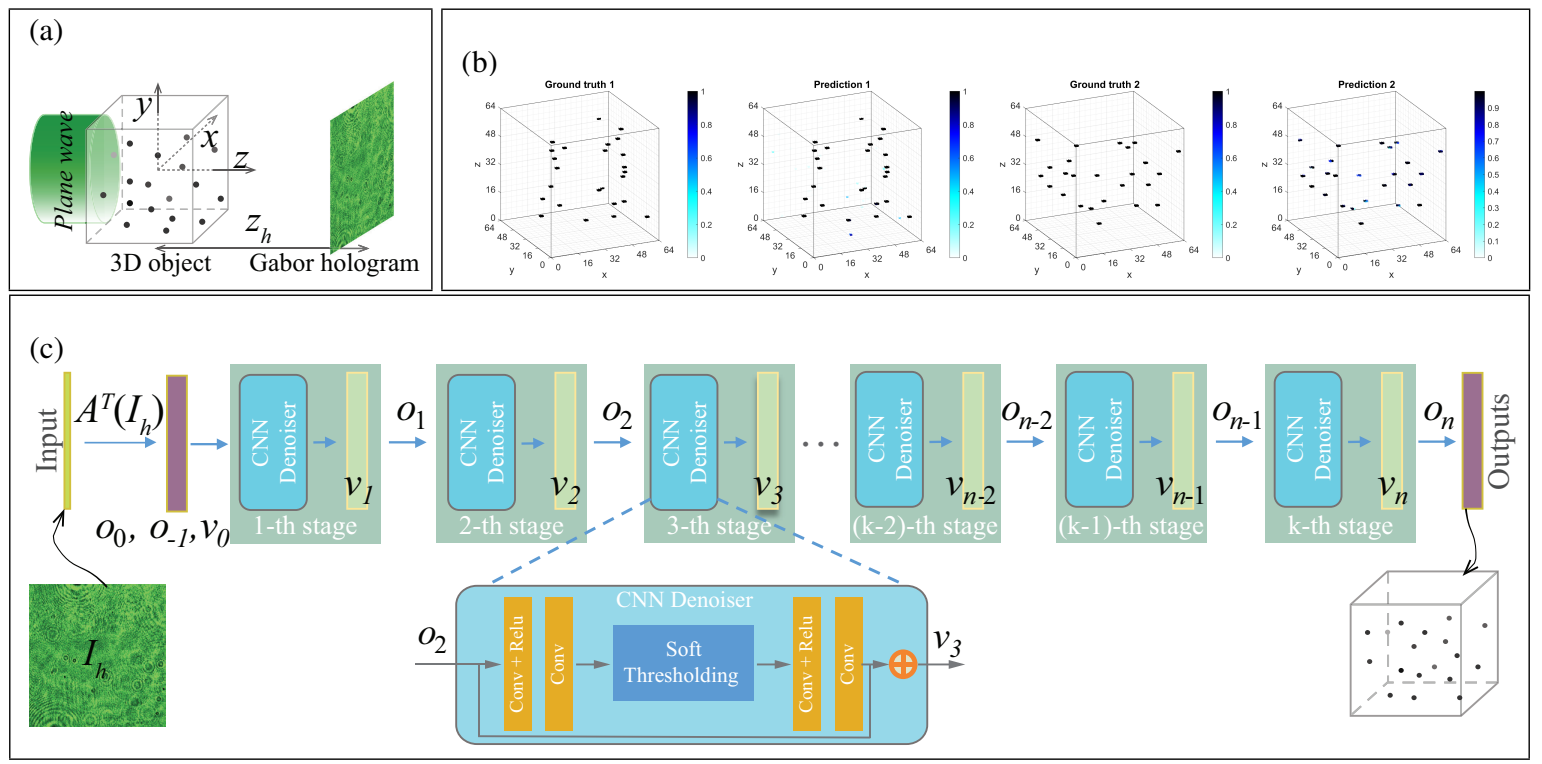

Fig. 1: (a) The scheme of the in-line holography system, (b) The results of the proposed method, and (c) the PBHolo-Net scheme. Here the input of the network is the captured holography image; the output is the restored 3D data; $o_{i}$ denotes the restored 3D object of the $i$-th stage; and $v_{i}$ denotes the auxiliary variable in the $i$-th stage. 
In an in-line holographic imaging system, as shown in Fig.(1)(a), a 3D object $o(\boldsymbol{r}, z)$ is illuminated by a plane wave of $\exp (j k z)$, where $\boldsymbol{r}=(x, y)$ and $k$ is the wave number. In the sensor plane, the propagated plane wave $u_{R}=$ $\mathscr{P}\left\{\exp (j k z), z_{h}\right\}$ and object wave $u_{O}=\mathscr{P}\left\{\exp (j k z) o(\boldsymbol{r}, z), z_{h}-z\right\}$ produce an interferometric pattern $I_{h}=\mid u_{O}+$ $\left.u_{R}\right|^{2}$, which is the hologram. $\mathscr{P}\left\{u(\boldsymbol{r}, z), z_{h}\right\}=u(\boldsymbol{r}, z) \otimes h\left(\boldsymbol{r}_{h}-\boldsymbol{r}, z_{h}-z\right)$ is the Rayleigh-Sommerfeld diffraction, and $h(\boldsymbol{r}, z)=\exp \left(j k \sqrt{\boldsymbol{r}^{2}+z^{2}}\right) / \sqrt{\boldsymbol{r}^{2}+z^{2}}$ is the free-space point spread function (PSF). The hologram $I_{h}$ can be re-write as $I_{h}=2 \mathfrak{R}\{\mathscr{A} o(\boldsymbol{r}, z)\}+1+\left|u_{O} u_{R}\right|^{2}$, where $\mathscr{A}$ is the forward wavefield propagation operator [2].

To cope with the problem, we proposed a deep PBHolo-Ne which is constructed by unrolling the Fast Iterative Shrinkage-Thresholding Algorithm(FISTA). FISTA [7] is an efficient proximal gradient method which can solve the inverse problem iteratively. In this problem, each iteration contains a update step to reduce error residuals of holography images and a denoising step to refine the restored 3D object. The scheme of the PBHolo-Net is shown in Figure 1, it consists of multiple stages, each of which corresponds to an iteration in FISTA. However, unlike FISTA method, all parameters(e.g. steplength, thresholds, etc) involved in PBHolo-Net are learned end-to-end via back-propagation; the network can learn nonlinear and sparsifying transforms to provide a more efficient and effective strategy for holography data restoration.

In each stage, a residual block consisting four convolution layers and a soft threshold layer is applied for noise removal with total variation regularization; and the reconstructed data is updated using an accelerated gradient descent algorithm. In the algorithm, the update step of $x_{i+1}$ in the $(i+1)$-th stage can be performed as $o_{i+1}=$ $o_{i}+\alpha_{i} \mathscr{A}^{T}\left(I_{h}-\mathscr{A}\left(o_{i}\right)\right)+\beta_{i}\left(v_{i+1}-o_{i}\right)+\gamma_{i}\left(o_{i}-o_{i-1}\right)$, where $\mathscr{A}()$ and $\mathscr{A}^{T}()$ is the forward and reverse model respectively, $\alpha_{i}, \beta_{i}$, and $\gamma_{i}$ denotes the learn able step lengths of the corresponding terms in the update step of the $i$-th stage. The total loss function is defined as the weighted sum of the mean square error loss of the 3D data and a symmetry penalty of the denoising network. Such loss design can guarantee both data fidelity and prior information fidelity.

\section{Results}

We have verified the feasibility of the proposed PBHolo-Net with numerically generated holograms of randomly distributed volumetric particles. The dimension of the volumetric particles are $64 \times 64 \times 5$, with a lateral of $10 \mu \mathrm{m}$ and axial resolution of $10 \mathrm{~mm}$. The particle concentration is $12.2 \times 10^{-3} \mathrm{ppp}$, while each particle is of single pixel size. In the hologram imaging system, the wavelength of the illumination is $\lambda=633 \mathrm{~nm}$, and the distance between the origin of the coordinate system and the hologram detection plane is $50 \mathrm{~mm}$.

Five hundred holograms, along with the 3D particle volumes, have been feed into the proposed PBHolo-Net to perform the training. Among them, four hundred holograms were used for training and the left was used for testing. The network contains only 8,424 trainable parameters, and all feature maps are generated with $3 \times 3$ kernels, which can prevent data overfitting. We have trained the model on a workstation with an NVIDIA GTX $1080 \mathrm{Ti}$ GPU for 100 epochs with a batch size of four, which took about 25 minutes for the whole training process. The final loss value of the testing reached to 0.005. The trained PBHolo-Net can perform hologram reconstruction in $5 \mathrm{~s}$, compared to about one minute with the conventional deconvolution method [2]. Two selected groups of the ground-truths and the corresponding predictions with the trained network are shown in Fig. 1(b). It can be seen that the locations of the particles have been reconstructed precisely, with only slight gray level degradation. It should be mentioned that the input of the network is only one single in-line hologram with the reference beam subtracted, which can be achieved conveniently by a single shooting in a real experiment.

\section{References}

1. D. Gabor, “A new microscopic principle," Nature 161, 777-778 (1948).

2. D. J. Brady, K. Choi, D. L. Marks, R. Horisaki, and S. Lim, “Compressive holography," Opt. Express 17, 13040-13049 (2009).

3. K. Mallery and J. Hong, "Regularized inverse holographic volume reconstruction for 3d particle tracking," Opt. Express 27, 18069 (2019).

4. Y. Rivenson, Y. Wu, and A. Ozcan, "Deep learning in holography and coherent imaging," Light. Sci. \& Appl. 8 (2019).

5. T. Shimobaba, T. Takahashi, Y. Yamamoto, Y. Endo, A. Shiraki, T. Nishitsuji, N. Hoshikawa, T. Kakue, and T. Ito, "Digital holographic particle volume reconstruction using a deep neural network," Appl. Opt. 58, 1900-1906 (2019).

6. S. Shao, K. Mallery, S. S. Kumar, and J. Hong, "Machine learning holography for 3D particle field imaging," Opt. Express 28, 2987-2999 (2020).

7. A. Beck and M. Teboulle, "A fast iterative shrinkage-thresholding algorithm for linear inverse problems," SIAM journal on imaging sciences 2, 183-202 (2009). 